\title{
Measuring Mindfulness: First Steps Towards the Development of a Comprehensive Mindfulness Scale
}

\author{
Claudia Bergomi $\cdot$ Wolfgang Tschacher $\cdot$ Zeno Kupper
}

Published online: 15 April 2012

(C) Springer Science+Business Media, LLC 2012

\begin{abstract}
The present study describes the development of and results obtained from the first version of a new mindfulness scale: the Comprehensive Inventory of Mindfulness Experiences beta (CHIME- $\beta$ ). The aim of the present analysis was to investigate two relevant open questions in mindfulness assessment: (1) the coverage of aspects of mindfulness and (2) the type of interrelationships among these aspects. A review of the aspects of mindfulness assessed by eight currently available mindfulness questionnaires led to the identification of nine aspects of mindfulness. The CHIME- $\beta$ was constructed in order to cover each of these aspects in a balanced way. Initially, principal component and confirmatory factor analyses, as well as reliability and validity analyses, were performed in the entire sample $(n=313)$ of individuals from the general population and mindfulness-based stress reduction (MBSR) groups. The factor structure that emerged from this analysis was further investigated in meditation-trained individuals $(n=$ 144) who had just completed an MBSR intervention. Results suggested a four-factor structure underlying the nine aspects proposed. The relationship between these mindfulness factors appears to be influenced by the degree of meditation experience. In fact, the mindfulness factors showed a greater interconnectedness among mediationtrained participants. Finally, data suggest that a nonavoidant stance plays a central role in mindfulness, while the capacity to put inner experiences into words may be related to mindfulness rather than a component of the construct.
\end{abstract}

\footnotetext{
C. Bergomi $(\triangle) \cdot$ W. Tschacher $\cdot$ Z. Kupper

University Hospital of Psychiatry, Department of Psychotherapy, University of Bern,

Laupenstrasse 49,

CH-3010 Bern, Switzerland

e-mail: bergomi@spk.unibe.ch
}

Keywords Mindfulness · Questionnaire · Self-report · Assessment

\section{Introduction}

Mindfulness has been described as a particular way of paying attention: on purpose, in the present moment, and nonjudgmentally (Kabat-Zinn 1994). During the last three decades, several mindfulness-oriented interventions, such as mindfulness-based stress reduction (MBSR; Kabat-Zinn 1990) and mindfulness-based cognitive therapy (MBCT; Segal et al. 2002; Teasdale et al. 1995), have been developed and their efficacy has been established in a number of studies (Grossman et al. 2004; Hofmann et al. 2010; Shigaki et al. 2006). There has been an increasing focus in current research on the ways in which mindfulness affects mental and physical health (Baer 2010; Coffey and Hartman 2008; Dimidjian and Linehan 2003; Shapiro et al. 2006). Thus, the availability of valid measures of the construct is crucial (Baer 2010; Shapiro et al. 2006). Correspondingly, in recent years, the assessment of mindfulness has received increasing attention. During the last decade, at least eight mindfulness questionnaires have been developed and validated: the Freiburg Mindfulness Inventory (FMI; Buchheld et al. 2001; Walach et al. 2006), the Mindful Attention Awareness Scale (MAAS; Brown and Ryan 2003), the Cognitive and Affective Mindfulness Scale-Revised (CAMS-R; Feldman et al. 2007; Hayes and Feldman 2004), the Southampton Mindfulness Questionnaire (SMQ; Chadwick et al. 2008, 2005, in Baer et al. 2006), the Kentucky Inventory of Mindfulness Scale (KIMS; Baer et al. 2004), the Five Facet Mindfulness Questionnaire (FFMQ; Baer et al. 2006), the Philadelphia Mindfulness Scale (PHLMS; Cardaciotto et al. 2008), and the Toronto Mindfulness Scale (TMS; Lau et al. 2006). 
Recently, self-report measures of mindfulness were developed for adolescents and children: the Child and Adolescent Mindfulness Measure (Greco et al. 2011) and an adaptation of the MAAS (Brown et al. 2011). Moreover, some interesting propositions and developments have been made with regard to methods of assessing mindfulness that do not rely on self-reports (for example Bishop et al. 2004; Burg and Michalak 2010; Collins et al. 2009; Dobkin 2008; Frewen et al. 2008).

The present paper will first describe relevant open questions relating to the conceptualization and operationalization of mindfulness. An overview of all aspects of mindfulness that have been proposed in previous operationalizations will be used as the basis for the construction of a preliminary version of a new mindfulness scale. Finally, results concerning the factor structure and validity of the preliminary scale will be presented.

\section{Are Current Mindfulness Scales Measuring the "Same} Mindfulness"?

An overview of the currently available mindfulness scales shows that the conceptualizations of mindfulness upon which they are based differ in several respects. The more evident point of divergence concerns which aspects of mindfulness are covered by the scales (Christopher et al. 2009). Semantic coverage ranges from the one-dimensional assessment of mindfulness as the direction of attention in the present moment (as in the MAAS), through twodimensional conceptualizations (e.g., PHLMS and TMS), to a multifaceted understanding of the construct encompassing aspects such as non-reactivity to experiences and the capacity to describe inner experiences (e.g., KIMS and FFMQ). In other words, while some questionnaires are based on a narrow conceptualization of mindfulness, others provide a broader semantic coverage of the construct.

Current scales not only vary widely in terms of the choice of aspects of mindfulness, but also in terms of the conceptualization of the relationship between these aspects. Results from scales such as the KIMS, FFMQ, and PHLMS support a conceptualization of mindfulness as being separable in aspects or components that are stable over different populations (Baer et al. 2004, 2006; Cardaciotto et al. 2008). Contrary to this, studies involving the CAMS-R, FMI, and SMQ favor a more holistic conceptualization of mindfulness as entailing interconnected aspects that cannot be meaningfully disentangled in order to create stable questionnaire subscales (Buchheld et al. 2001; Chadwick et al. 2008; Feldman et al. 2007).

Current mindfulness scales also vary with respect to the trait-to-state continuum. The TMS assesses the capacity to invoke a mindfulness state during a very short period of time, for instance, during a meditation session. The FMI,
MAAS, and PHLMS measure mindfulness as a quasi-trait, as respondents are required to refer to the items over a period of time, for instance, the last two weeks. In the KIMS, CAMS-R, and FFMQ, respondents are asked to rate whether items are generally true for them, thus treating mindfulness as a trait (Cardaciotto 2005).

In sum, current mindfulness scales base on conceptualizations of mindfulness that differ over relevant issues. Some differences may be desirable. For example, as trait mindfulness and state mindfulness are two related but different constructs (e.g. Thompson and Waltz 2007), it is suitable to possess scales allowing for the measurement of each of them. Nevertheless, the open questions related to (1) the coverage of aspects of mindfulness and (2) the kind of relationships between these aspects pose relevant problems. They hamper a meaningful comparison of the results from different studies and point to a lack of consensus regarding the conceptualization of mindfulness (Brown et al. 2007; Dimidjian and Linehan 2003; Hayes and Wilson 2003; Malinowski 2008).

\section{Present Research}

The present study is of exploratory nature. Its aim was to contribute to the development of a new measure of mindfulness, the Comprehensive Inventory of Mindfulness Experiences (CHIME). First, a theoretical integration of previous operationalizations will be proposed and used to construct a preliminary version of the scale, the CHIME- $\beta$. On the basis of the CHIME- $\beta$ the two open questions described above will be explored: (1) appropriate coverage of the components of mindfulness and (2) the nature of the relationship between the components covered by the scale. Pertaining to the first issue, the factorization of the CHIME- $\beta$ and the correlations among the components may shed light on the centrality of the aspects of mindfulness included in the analysis and designate the aspects that are more (or less) central to the construct. The second issue (i.e., the interrelationship between the aspects of mindfulness) may be addressed through model comparison, as well as by the stability of factor solutions in populations with different meditation experiences. A stable solution would speak for the possibility to separate mindfulness in stable components while an instability in the factorization would support a holistic understanding of mindfulness.

\section{Method}

Scale Construction

\section{A Review of Previously Proposed Aspects of Mindfulness}

In 2004, Bishop and colleagues made an important contribution to the field by proposing a consensual operational 
definition of mindfulness that distinguishes between two main components: (1) self-regulation of attention so that it is directed in the present moment and (2) a particular orientation involving curiosity, openness, and acceptance. In this section, all aspects of mindfulness included in the eight validated mindfulness questionnaires available as of 2009 were reviewed and grouped according to their content. For this purpose, the two-component conceptualization proposed by Bishop and colleagues was used as a guideline, beginning with aspects pertaining to self-regulation of attention, followed by aspects describing a mindful orientation. For all scales, the aspects of mindfulness included in the following review correspond to the aspects indicated by the scale's authors in the validation studies. As the FMI is shown to have a different component structure across different populations, the aspects of mindfulness measured by this scale will be drawn not only from the results of the validation study (Walach et al. 2006) but also from two studies in which the FMI was subjected to principal component analysis (PCA). In one study, PCA used in an undergraduate college population $(N=196)$ yielded three components: acceptance and openness to self and experience, mind/body awareness, and non-attachment to thoughts (Leigh et al. 2005). In the other study, PCA in 409 subjects from the general population provided four interpretable components: non-avoidant awareness, non-reactivity to negative experiences, self-acceptance, and concentration (Bergomi 2007).

Self-regulation of attention is generally regarded as the central element of mindfulness (Bishop et al. 2004; KabatZinn 1994). Within self-regulation of attention, two distinct aspects have been described and assessed: the first may be labeled observing, attending experiences and the second, acting with awareness. Observing, attending experiences refers to the directing of one's attention to present experiences, including one's current thoughts, feelings, sensations, and perceptions. This aspect is included in the CAMS (factor: awareness), FMI (mindful presence in Walach et al. 2006; mind/body awareness in Leigh et al. 2005), KIMS (observing), FFMQ (observe), and the PHLMS (awareness). The second aspect of attentional self-regulation, acting with awareness, addresses focusing on one thing at a time or concentrating (i.e., not running on automatic pilot) while doing things. This aspect is assessed in the MAAS (presence), KIMS (acting with awareness), FFMQ (actaware), FMI (concentration in Bergomi 2007), and CAMS (attention and present-focus).

With regard to the second component of mindfulness, the literature is less clear as to exactly which aspects it should comprise. According to the popular definition by KabatZinn (1994), nonjudgment of experiences and self is a central aspect of a mindful orientation. It means accepting or welcoming one's own feelings, thoughts, sensations, and perceptions without being adversely judgmental or critical. Nonjudgmental acceptance is well represented among the mindfulness scales: it is measured by the KIMS (accepting without judgment), FMI (nonjudgmental acceptance in Walach et al. 2006; acceptance and openness to self and experience in Leigh et al. 2005; self-acceptance in Bergomi 2007), FFMQ (nonjudge), and SMQ (accepting difficult thoughts/images and oneself versus judging cognitions and self). Most of the acceptance items focus on acceptance of one's own experiences, such as thoughts and feelings (KIMS16: I believe some of my thoughts are abnormal or bad and I shouldn't think that way). However, many of the acceptance-related items, particularly those from the FMI, tap a semantically different aspect: an accepting, compassionate stance that is more directly related to oneself (FMI19: I accept myself as I am). These two aspects, nonjudgment of experiences and self-acceptance, are clearly overlapping but semantically distinguishable. This distinction is supported by empirical evidence: Baer et al. (2006) subjected all the items of five mindfulness scales (the MAAS, FMI, SMQ, KIMS, CAMS) to exploratory factor analysis (EFA), which led to the construction of the FFMQ (Baer et al. 2006). In their analysis, none of the FMI acceptance items loaded on the nonjudgment of experience factor. Therefore, in the present study, nonjudgment of experiences and self-acceptance were treated as two distinct aspects of mindfulness.

The acceptance scale of the PHLMS does not directly capture self-acceptance or nonjudgment but rather addresses experiential avoidance (PHLMS16: If there is something I don't want to think about, I'll try many things to get it out of my mind). The term "experiential avoidance" refers to behaviors aimed at altering the form and frequency of particular private experiences (e.g., memories, thoughts, bodily sensations, emotions) in order to avoid them (Hayes et al. 1996). As pointed out by Bishop et al. (2004), a mindful orientation to experiences is characterized by openness and curiosity - in other words, by a willingness and readiness to expose oneself to (pleasant and unpleasant) experiences (i.e., the opposite of experiential avoidance). Among the current scales, this aspect is not only addressed by the PHLMS, but is also found in the FMI (openness to experience in Walach et al. 2006; non-avoidant awareness in Bergomi 2007) and the SMQ (allowing attention to remain with difficult cognitions versus experiential avoidance). Moreover, the curiosity scale in the TMS captures a construct closely related to the willingness to expose oneself to experiences.

A further proposed aspect of mindfulness is non-reactivity to experience, which means refraining from impulsive reactions to experiences. Non-reactivity to experience contributes to a disruption of automatic reaction patterns. Baer et al. (2006) suggested that this capacity "may be seen as [a] 
way(s) of operationalizing acceptance" (p. 42). This aspect of mindfulness is captured by the FFMQ (nonreact), FMI (non-reactivity to inner experience, Bergomi 2007), and SMQ (letting difficult cognitions pass without reacting versus rumination/worry).

The acceptance scale of the CAMS could not be clearly assigned to one of the proposed aspects. This scale involves three items: CAMS3: I can tolerate emotional pain; CAMS4: I can accept things I cannot change; and CAMS10: I am able to accept the thoughts and feelings I have. Semantically, these items can be subsumed into the following components: nonjudgment/acceptance of experiences, non-reactivity to inner experiences, and willingness and readiness to expose oneself to (pleasant and unpleasant) experiences.

The TMS, FMI, and SMQ address aspects of mindfulness that are related to insight rather than to an accepting attitude: decentering (TMS), mindful presence (FMI, Walach et al. 2006), non-attachment to thoughts (FMI, Leigh et al. 2005), insight (FMI, Walach et al. 2006), and decentered awareness (SMQ). When analyzed at the item level, these subscales seem to comprise two distinct aspects: on one side, non-identification with own experiences (TMS40: I was aware of my thoughts and feelings without over-identifying them) and on the other side, insightful understanding (FMI16: I see how I create my own suffering). Of the two, non-identification with own experiences is more processrelated and refers to the act of experiencing one's thoughts and feelings from a decentered perspective, without overidentifying with them or elaborating them further. This aspect of mindfulness has also been described as metacognitive insight or "experiencing thoughts as thoughts (that is, as events in the mind, rather than as direct readouts on reality)" (Teasdale 1999, p. 147). Insightful understanding means understanding thoughts and feelings from a broader perspective, being aware of their relativity and caducity, and gaining insight into the inner workings of the mind.

Finally, describing/describe (KIMS, FFMQ) has also been suggested as a component of mindfulness. Describing or labeling refers to the ability to put feelings, mood, perceptions, and thoughts into words. The inclusion of this capacity as a component of mindfulness in self-report measures was first proposed by Baer et al. $(2004,2006)$ and was based largely on the conceptualization of mindfulness proposed by dialectical behavioral therapy (DBT; Linehan 1993).

In sum, a review of the mindfulness literature suggests a working hypothesis list of nine aspects of mindfulness (Table 1): (1) observing, attending to experiences (OBSERVE), (2) acting with awareness (ACTAWARE), (3) nonjudgment/acceptance of experiences (NONJUDGE), (4) self-acceptance (SELFACCEPT), (5) willingness and readiness to expose oneself to experiences/non-avoidance (NONAVOID), (6) non-reactivity to experience (NONREACT), (7) non-identification with own experiences (NONIDENTIFY), (8) insightful understanding (INSIGHT), and (9) labeling/describing (DESCRIBE). Given the complexity and richness of the mindfulness construct, the aspects of mindfulness found in the literature could have been classified in a different manner. The scope of the arrangement presented here is not intended to suggest a list of nonoverlapping or independent (orthogonal) aspects of mindfulness, but rather to provide one possible meaningful description of all components of the construct. This list will allow a testing of hypotheses concerning the structure of mindfulness.

\section{The Construction of the CHIME- $\beta$}

The CHIME- $\beta$ construction was based on the list of mindfulness aspects derived from the above review of all published scales. New items were formulated instead of relying on already existing ones in order to avoid a biased selection of items. Nevertheless, previous formulations were largely taken into account in the development of the items of the CHIME- $\beta$. For each aspect, seven to nine items were created by the authors, two of whom have personal, long-term experience with mindfulness meditation and Buddhist psychology. From this initial item pool, four items per aspect were selected through consultation with meditation-naïve individuals. Thus, each aspect was represented in a balanced way in the final item pool to avoid certain aspects from being represented more than others and thus more strongly influencing the results of dimension reduction analysis. Moreover, the procedure aimed at selecting items that a general population sample would easily understand. The resulting scale, the CHIME- $\beta$, comprised 36 items covering the previously proposed nine aspects of mindfulness with four items each. The current project regards mindfulness as a general human capacity occurring in daily life that is susceptible to change, for example, through specific training (Brown and Ryan 2004; Buchheld et al. 2001). Hence, mindfulness was conceptualized as a quasi-trait (Cardaciotto 2005). Thus, the CHIME- $\beta$ instructs respondents to relate items to the past seven days of their life. Participants respond using a six-point Likert scale ( $1=$ applies fully to 6=does not apply at all).

\section{Participants}

The study sample comprised 313 participants, 128 from the general population, and 185 from MBSR groups. Participants from the general population completed a questionnaire once while participants from the MBSR groups were asked to complete a questionnaire at the beginning of the eight-week intervention (between group sessions one and two) and at the end of the intervention (during the week before the final session). Demographic data are shown in Table 2. A subsample of 68 participants from the general population also completed an additional mindfulness measure, the FFMQ. The 
Table 1 Overview of eight mindfulness questionnaires and the mindfulness aspects they include

Aspects of mindfulness

OBSERVE ACTAWARE NONJUDGE SELFACCEPT NONAVOID NONREACT NONIDENTIFY INSIGHT DESCRIBE

\begin{tabular}{lllllll}
\hline MAAS & & $\mathrm{x}$ & & & & \\
FMI & $\mathrm{x}$ & $\mathrm{x}$ & $\mathrm{x}$ & $\mathrm{x}$ & $\mathrm{x}$ & $\mathrm{x}$ \\
KIMS & $\mathrm{x}$ & $\mathrm{x}$ & $\mathrm{x}$ & & $\mathrm{x}$ & $\mathrm{x}$ \\
FFMQ & $\mathrm{x}$ & $\mathrm{x}$ & $\mathrm{x}$ & $\mathrm{x}$ & $\mathrm{x}$ & \\
CAMS & $\mathrm{x}$ & $\mathrm{x}$ & $\mathrm{x}$ & $\mathrm{x}$ & $\mathrm{x}$ & $\mathrm{x}$ \\
SMQ & & & $\mathrm{x}$ & & $\mathrm{x}$ \\
PHLMS & $\mathrm{x}$ & & $\mathrm{x}$ & & $\mathrm{x}$ \\
TMS & & & & \\
\hline
\end{tabular}

For each row crosses indicate the aspects of mindfulness that are included in the respective questionnaires

participants' education levels ranged from 10 school years or less $(2.9 \%)$ to university or advanced technical college degree $(37.0 \%)$. The majority of the participants $(39.0 \%)$ reported diploma or apprenticeship as their highest educational qualification. Seventy-nine participants reported practicing meditation on a regular basis (at least one session per week); this included meditation techniques in a broad sense, such as Yoga, autogenic training, progressive muscle relaxation, Zen meditation, Vipassana meditation, Qigong, and Tai Chi. Ethnic information was not collected, but the sample was predominantly Caucasian. The sample was nonclinical as it included individuals from the general population participating in MBSR groups that focused on teaching a more mindful and conscious way of coping with everyday stress. The mean psychopathological symptom load of all groups was in the nonclinical range (i.e., within one standard deviation of the mean scale $t$ score). This was also true for the mean psychopathological symptom load among participants from MBSR groups at the beginning of the intervention (GSI: mean=58.2, SD=12.5, $n=185$ ). The MBSR interventions took place in several cities in German-speaking Switzerland and were conducted by teachers from the MBSR Union Switzerland.

Table 2 Demographic data

\begin{tabular}{llllll}
\hline & $N$ & $\%$ female & $\begin{array}{l}\text { Mean age, } \\
\text { SD }\end{array}$ & $\begin{array}{l}\text { \% Meditation } \\
\text { practice }\end{array}$ & GSI, SD \\
\hline $\begin{array}{l}\text { Entire sample } \\
\begin{array}{l}\text { Subsample } \\
\text { with FFMQ }\end{array}\end{array}$ & 68 & $43.1(0)$ & $37.4,11.2(0)$ & 25.0 & $50.1,10.1$ \\
$\begin{array}{c}\text { Meditation- } \\
\text { trained }\end{array}$ & 144 & $68.8(0)$ & $45.0,9.7(1)$ & 25.7 & $52.1,11.8$ \\
\hline
\end{tabular}

The numbers of missing cases are in parentheses

Meditation practice current meditation practice, including meditation techniques in a broad sense, at least one session per week; GSI Global Symptom Index $t$ value (psychopathological symptom load)
Two analyses were performed using this sample of participants. The first analysis used the data of the entire sample. For this analysis, the initial measurement in the MBSR groups was used. Thus, data in the first analysis involved largely meditation-untrained individuals. The second analysis considered 144 participants who had completed the questionnaire at the end of the MBSR interventions. Participants in this sample had thus just undergone an intensive 8-week mindfulness-based intervention comprising a theoretical introduction to mindfulness and daily practice of approximately $1 \mathrm{~h}$ and were thus all trained in meditation.

\section{Instruments}

Participants completed the CHIME- $\beta$, the German version of the Brief Symptom Inventory (BSI, Franke 2000), and two scales of the Emotion-Regulation Skills Questionnaire (ERSQ; Berking and Znoj 2008). A subgroup of the general population $(N=68)$ also completed the German version of the Five Facets of Mindfulness Questionnaire (FFMQ; Heidenreich et al. 2011). Mindfulness was assessed using the CHIME- $\beta$, which was described above.

Psychological distress was measured using the BSI, which includes 53 items and measures subjective impairment due to somatic and psychological symptoms. The scale covers nine symptom dimensions (somatization, obsession-compulsion, interpersonal sensitivity, depression, anxiety, hostility, phobic anxiety, paranoid ideation, psychoticism) and includes a general severity index (GSI). Participants rated the items on a scale from 0 (not at all) to 4 (extremely often). In support of the validity of the scale, the GSI has been shown to be negatively associated with quality of life and with social support and it showed discriminant validity from personality measures (Franke 2000)

The ERSQ is a self-report instrument that utilizes a fivepoint Likert-type scale $(0=$ not at all to $4=$ almost always $)$ to 
assess adaptive emotion-regulation skills. In this study, two of the nine skills covered by the scale (each consisting of three items) were assessed: readiness to confront distressing situations (e.g., I did what I had planned, even if it made me feel uncomfortable or anxious) and modification (e.g., I was able to influence my negative feelings). The validity of these ERSQ subscales is supported by several results: in both community (Berking and Znoj 2008) and clinical (Berking et al. 2008) samples the two subscales used in this study were positively associated with measures of mental health and well-being and negatively with measures of psychopathology.

The FFMQ is a 39-item scale that measures five facets of mindfulness: non-reactivity to inner experience, observing/ noticing/attending to sensations/perceptions/thoughts/feelings, acting with awareness/automatic pilot/concentration/ nondistraction, describing/labeling with words, and nonjudgment of experience. Participants responded on a five-point Likert-type scale $(1=$ never or very rarely true to $5=$ very often or always true). The FFMQ was chosen because of its wide assessment of the mindfulness construct and the availability of subscales. The questionnaire was shown to have satisfying convergent and discriminant validity (Heidenreichet al. 2011).

All scales showed good reliabilities in this study's sample. In the entire sample the Cronbach's alpha were $\alpha=0.96$ for the BSI, $\alpha=0.83$ for the ERSQ subscale readiness to confront distressing situations and $\alpha=0.76$ for the ERSQ subscale modification. In the subsample to which the FFMQ was administered Cronbach's alpha for this scale was, with $\alpha=0.91$, highly satisfactory.

\section{Procedure}

MBSR teachers from the MBSR Union Switzerland distributed the questionnaires to their group participants, who participated voluntarily in the study. The study targeted 29 MBSR groups and mean participation was $74 \%$ (range= 25-100\%). Participants from the general population were solicited by the authors from among their friends and acquaintances. Participation in this case was also voluntary and the study followed guidelines of research ethics. Participants completed the questionnaires either on an online platform (51.4\%) or using paper and pencil. Several studies have shown that results obtained using online data collection techniques are typically consistent with those obtained through traditional methods (Gosling et al. 2004). The questionnaires were presented in the same order for each participant, with the exception of 68 participants, to whom the FFMQ was also administered and where the sequence of the FFMQ and CHIME- $\beta$ was counterbalanced. No compensation was paid for participation.
Statistical Procedures

As the CHIME- $\beta$ was developed for use in the general population, PCA was first conducted on the data of the entire sample, which included individuals largely untrained in meditation $(N=313)$. Before conducting PCA, the item distributions were screened. The overall reliability was calculated using Cronbach's alpha and the corrected item-total correlations were analyzed. Items with low $(r<0.20)$ corrected item-total correlation (Everitt 2002) were screened in order to establish if low item-to-scale correlation was due to a poor formulation of the items. The items retained after this preliminary analysis were subjected to PCA with oblique rotation (oblimin), which allows for correlations between the components. In contrast to EFA methods (e.g., principal axis factoring, maximum likelihood), PCA allows keeping as much variance as possible from the measured variables, since principal components are calculated using the total variance of the measured variables (Jolliffe 2002; Park et al. 2002). Although models resulting from PCA and EFAs can differ, when they do for a given set of data, then the number of principal components required for an adequate dimension reduction is equal or larger than the number of factors proposed by EFA (Jolliffe 2002). This is usually due to larger item loadings in the PCA solution (Jolliffe 2002) and thus to the formation of additional components (rather than to the further partitioning of factors into smaller components). This property of PCA is desirable for the present analysis. In fact, the aim was to reduce the nine aspects proposed above maintaining as much as possible of their dimensionality (i.e. not excluding too many) in order to study their interrelationships. Moreover, given the exploratory nature of the study, no a priori structure was expected to emerge from the analysis, which is also in line with use of PCA (Costello and Osborne 2005). In fact, the eight aspects of mindfulness covered in the CHIME- $\beta$ were not expected to emerge from the analysis as distinct factors but rather to merge into a smaller set of variables. The number of factors to be retained was determined using parallel analysis and Velicer's minimum average partial (MAP) test (O'Connor 2000). Parallel analysis gives the number of components accounting for more variance than the components derived from random data. The MAP test is based on the relative amount of systematic and unsystematic variance remaining in the correlation matrix after successive extractions of components (O'Connor 2000). Only items with a minimum loading of 0.40 on at least one factor (pattern matrix) were retained. Moreover, items that could not be clearly assigned to one factor (difference between absolute values of the highest and next highest loading below 0.20 ) were excluded from the final solution. Cross-comparison with loadings from the structure matrix was performed. 
The model derived from PCA was subjected to CFA. The aim of the CFA in the entire sample was not to confirm the generalizability of the model derived from the PCA, as this could only be done performing CFA in new samples (Cudeck and Browne 1983). Rather, the aim was to focus on analyzing the interrelationships of the components, i.e. comparing correlation and hierarchical models, performing correlation analysis at the level of latent variables (which limits the impact of measurement error; Tomarken and Waller 2005) or calculating regression coefficients to a putative higher-order mindfulness factor. In the CFA, accuracy of the model fit was tested with four fit indices: the Chi-square, the standardized root mean square residual (SRMR), the root mean square error of approximation (RMSEA), and the comparative fit index (CFI). Estimates were calculated using the maximum likelihood (ML) method. All items included in the analysis had skewness and kurtosis below 1, which is well below the cutoff values of 2 for skewness and 7 for kurtosis, suggested by West et al. (1995) for the use of the ML method. The fit of the models was evaluated using the following fit index cutoffs indicating a good fit: RMSEA $\leq$ 0.08 , SRMR $\leq 0.10$, and $\mathrm{CFI} \geq 0.90$ (Brown 2006; Browne and Cudeck 1993; Marsh et al. 2004; Schermelleh-Engel et al. 2003). More restrictive goodness of fit indices can be more easily achieved when latent variables are derived from item parcels (i.e., means of aggregated items pertaining to the same factor) but are too restrictive if latent variables are derived directly from the scale items (Marsh et al. 2004). Several authors have criticized the use of parcels in the scale development process, as parcels may hide possible model misspecifications and existing relationships among items and factors, thus hampering analysis of construct validity (Bandalos and Finney 2001; Cattell 1974; Christopher et al. 2009; Marsh et al. 2005). We therefore opted for analysis at the item level with less restrictive cutoffs.

Several models were tested with CFA: (1) a single-factor model in which one overall mindfulness factor directly accounts for the variance of all the indicators (i.e., items); (2) a correlational multiple-factor model representing the factor structure found in the PCA and in which the factors are allowed to freely correlate with one another; and (3) a hierarchical multiple-factor model in which an overall mindfulness construct explains the variance in the factors emerging from the PCA. Models were compared by means of Chisquare difference tests. If lack of fit was present in the best fitting model, modification indices were examined. Modification indices give an estimate of the expected Chi-square decrease (i.e., gain of model fit) if a particular parameter is left unconstrained. CFA was repeated in meditation-trained individuals. The 5:1 criterion for the proportion of sample size to number of items $(5 \times 28=140)$ suggested for CFA (Kline 2005) was, with $n=144$, fulfilled. Reliabilities were calculated with Cronbach's alpha. For correlational analysis, the Pearson product-moment correlation and partial correlations were used. Group differences were tested by means of t-tests. Reliability analysis, correlational analysis, and PCA were performed using the software package PASW Statistics 18. CFA was performed using Mplus 6.

Missing values in the questionnaire data from CHIME- $\beta$, FFMQ, BSI and ERSQ were screened. Missing values amounted to less than $0.5 \%$ of all cases. Possible biases due to missing values were evaluated by comparing results obtained with an iterative Markov Chain Monte Carlo (MCMC) multiple imputations procedure, mean substitution and pairwise deletion. As SPSS 18.0 cannot compute the statistical analysis required in this study (e.g., reliabilities and data reduction analysis) by pooling multiple data sets with imputed values (Acock 2005; Graham 2009), this strategy was not used. Instead, results based on the dataset computed by mean substitution were compared to both results based on five datasets computed with multiple imputation and results based on pairwise deletion. Results with mean substitution were highly congruent with results derived with the other strategies, e.g. in PCA $89 \%$ of the loadings on the pattern matrix deviated maximally by 0.01 (and no loading deviated by more than 0.03 ). This suggests that data derived through mean substitution are unbiased in the current dataset. Thus, data imputed by mean substitution were utilized for the analysis in both SPSS and MPLUS.

\section{Results}

Exploring the Structure of Mindfulness

The following analyses were performed in the entire sample $(N=313)$. Cronbach's alpha over all 36 items was very high ( $\alpha=0.94)$, but four items showed corrected item-total correlations below 0.20 . One item (I am the greatest critic of myself) pertained to the NONJUDGE aspect; the second item (I am right in the middle of my thoughts), to the NONIDENTIFY aspect; the third (In distressing situations, I feel myself inwardly distressed), to the NONREACT aspect; and the fourth (I know that my experiences are transient), to the INSIGHT aspect of mindfulness. A closer analysis of these items suggested that they may have been ambiguous and may thus have been interpreted differently by participants, leading to the observed lack of association. The four items were excluded from consequent analysis, and the remaining 32 items were again subjected to reliability analysis. The ensuing Cronbach's alpha was 0.95 , and all corrected item-total correlations were above 0.20 .

The 32 mindfulness items were subjected to PCA. The Bartlett Test of Sphericity was significant $(\mathrm{CHI}=5,974.0$, 
$p<0.001)$, and the KMO coefficient was very good $(>0.90)$. The number of participants $(N=313)$ was large enough to produce a stable solution even in the case of low item communalities or low determination of the components (MacCallum et al. 1999). In the present data, the MAP test indicated a four-component solution. Parallel analysis showed that three components had eigenvalues greater than those of the components derived from random data. The four-component solution was retained because specifying too few components is potentially more harmful than specifying too many as it might lead to a loss of important information (Zwick and Velicer 1986). Moreover, the scree plot also indicated a fourcomponent solution. The chosen four-component solution accounted for $57.2 \%$ of the total variance. As shown in Table 3, 28 of the original 32 items met the inclusion criteria. Of the four items excluded, one showed the highest loading below 0.40 , while three did not show the required minimal loading difference of 0.20 between the two highest loadings. Cross-comparison of the pattern and the structure matrices support this structure. As expected due to the associations between the components, loadings in the structure matrix were larger, leading to numerous cross-loadings. Nevertheless, for each variable the highest pattern coefficient coincided with the highest structure coefficient. Moreover, for each component, the items showing the highest pattern coefficients were the same as those having the highest structure coefficients.

The component accounting for most variance comprised items from SELFACCEPT (3 items of the 3 retained in the final solution), NONJUDGE (4/4), NONREACT (2/3), INSIGHT (2/3), NONIDENTIFY (1/3), and NONAVOID (1/4). This component describes an accepting, nonreactive, and insightful orientation. As was expected, one component addressed self-regulation of attention and included items from OBSERVE (3/4) and ACTAWARE (3/4). It was termed present awareness. The third component included all four DESCRIBE items and was thus named describing of experiences. The fourth component included mostly NONAVOID items (3/

4) but also an OBSERVE and a NONIDENTIFY item, thus capturing an open, non-avoidant orientation.

The component structure from the PCA was further examined in a CFA (Table 4). As expected, the single-factor model showed a poor fit, which indicates that the structure of the CHIME- $\beta$ is not unidimensional. The correlational four-factor model representing the factor structure found in the PCA yielded good results except for the CFI, which was slightly below the cutoff value of 0.90 . Finally, the hierarchical four-factor model was tested. It was compared with the correlational four-factor model by means of Chi-square difference. The hierarchical model yielded a significantly higher Chi-square value, which supports the correlational model. Nevertheless, CFI, SRMR, and RMSEA showed only slight differences, which speaks against rejection of the more parsimonious hierarchical model.
According to the modification indices for the correlational four-factor model (i.e., the best fitting model), the lack of fit was primarily due to existing correlations between items from the accepting, nonreactive, and insightful orientation factor, not accounted for in the model. As this suggested that this factor may be meaningfully subdivided into further subscales, PCA was run on its 13 items. Parallel analysis pointed to a one-component solution, while MAP indicated the presence of two components. PCA enforcing a twocomponent solution accounted for $61.7 \%$ of total variance. The first component comprised five items from the mindfulness aspects: SELFACCEPT (item 42), NONREACT (item 34), NONIDENTIFY (item 7), INSIGHT (item 29), and NONAVOID (item 30). The second component comprised the four NONJUDGE items. The four remaining items did not show differences above 0.20 between their loadings on the two components. This suggests that within the accepting, nonreactive, and insightful orientation items, those relating to a nonjudgmental stance tend to emerge as a meaningful distinct component, even if this tendency was not strong enough to show in the analysis at the level of the entire item pool. Interestingly, SELFACCEPT, NONREACT, INSIGHT, NONIDENTIFY, and NONAVOID showed a high degree of interconnectedness, as items referring to these aspects tended to load on the same component.

\section{Reliability, Validity, and Correlational Analyses}

In order to test the reliability of the proposed mindfulness factors and of the overall scale, Cronbach's alphas were calculated for each. All alpha coefficients were very satisfactory ( $\alpha \geq 0.85$ ), with the exception of the factor open, non-avoidant orientation $(\alpha=0.65)$. This suggests a larger heterogeneity of the items in this factor and is acceptable for a factor including only five items (John and Benet-Martinez 2000; Ryff and Keyes 1995). Discriminant and convergent correlations between each of the four factors, the assessed emotionregulation skills, and psychopathological symptom load were also calculated (Table 5, top). All correlations showed the expected pattern of mindfulness to be positively associated with emotion-regulation skills and negatively associated with psychopathological symptom loads. Among the mindfulness factors, accepting, nonreactive, and insightful orientation showed the strongest associations with lack of symptoms and regulatory skills. Finally, correlations between the FFMQ and CHIME- $\beta$ (criterion validity) were calculated (Table 5, bottom). The correlations indicate the validity of the CHIME$\beta$. In fact, correlations between the overall scores were high and each FFMQ subscale had the strongest correlation with its semantically closest CHIME- $\beta$ factor. In order to examine whether the CHIME- $\beta$ provides incremental validity over an existing mindfulness scale, the FFMQ, partial correlations were computed. First, correlations between CHIME- $\beta$, BSI 
Table 3 Component structure of CHIME- $\beta$ in the entire sample

Component loading

Item

$1 \quad 2 \quad 3$

4

Factor 1: accepting, nonreactive and insightful orientation

20. (SELFACCEPT) I can accept myself as I am.

38. (NONJUDGE) I believe my thoughts are abnormal and tell myself that I should not be

thinking like that.

27. (NONJUDGE) I am ashamed because of my thoughts.

42. (SELFACCEPT) Even when I see my flaws, I can still be friendly towards myself.

05. (NONJUDGE) I think that my feelings are bad or inappropriate and that I should not have them.

09. (SELFACCEPT) I have an appreciative attitude towards myself.

34. (NONREACT) Even in painful and problematical situations, I can inwardly stay calm and serene.

16. (NONJUDGE) I judge my thoughts and feelings as being good or bad.

07. (NONIDENTIFY) I can distance myself from my thoughts and observe them from another angle.

29 (INSIGHT) When I see how I create big problems from small difficulties, I can smile about it.

40. (INSIGHT) I can consider things from different perspectives.

30. (NONAVOID) I can confront unpleasant situations as well.

01. (NONREACT) I notice my feelings, without having to immediately put them into action.

$\begin{array}{lrrr}\mathbf{0 . 8 0 9} & -0.011 & 0.093 & -0.056 \\ \mathbf{0 . 7 7 7} & -0.211 & 0.160 & 0.175 \\ \mathbf{0 . 7 6 1} & -0.216 & 0.117 & 0.171 \\ \mathbf{0 . 7 6 0} & 0.148 & -0.080 & -0.067 \\ \mathbf{0 . 7 1 7} & -0.192 & 0.173 & 0.201 \\ \mathbf{0 . 6 6 0} & 0.071 & 0.117 & -0.026 \\ \mathbf{0 . 6 4 9} & 0.350 & -0.067 & -0.304 \\ \mathbf{0 . 6 0 6} & -0.043 & 0.072 & -0.020 \\ \mathbf{0 . 5 8 7} & 0.218 & -0.053 & 0.158 \\ \mathbf{0 . 5 7 5} & 0.106 & 0.023 & 0.103 \\ \mathbf{0 . 5 4 0} & -0.100 & 0.157 & 0.300 \\ \mathbf{0 . 4 8 5} & 0.101 & 0.235 & 0.102 \\ \mathbf{0 . 4 3 3} & 0.172 & 0.151 & 0.144\end{array}$

Factor 2: Present awareness

02. (OBSERVE) When I wash my hands or brush my teeth I notice my movements and the sensations occurring in my body.

13. (OBSERVE) During daily activities as well, I pay attention to the sensations in my body.

03. (ACTAWARE) While I am doing something I pay attention to how I do it.

35. (OBSERVE) When I eat, I consciously pay attention to the taste of the food.

36 (ACTAWARE) I find it difficult to pay attention to the "here and now" and to concentrate on that which currently happens.

14. (ACTAWARE) I rush through my activities without paying much attention to them.

$-0.088$

$-0.063$

0.052

0.034

0.238

0.152

$\mathbf{0 . 5 3 6} 0.072$

$\mathbf{0 . 7 9 5} 0.023$

0.109

$\mathbf{0 . 7 4 4} \quad 0.017$

0.166

$\mathbf{0 . 6 3 2} 0.147$

0.140

$\begin{array}{lll}\mathbf{0 . 6 1 0} & 0.199 & 0.117\end{array}$

$\mathbf{0 . 5 6 5}-0.111-0.205$

0.262

Factor 3: Describing of Experiences

04. (DESCRIBE) I have trouble finding the right words to express my feelings.

\subsection{3}

0.004

0.890

$-0.043$

$-0.059$

0.039

0.882

$-0.043$

0.003

0.138

$0.796 \quad 0.034$

0.217

0.014

$\mathbf{0 . 7 0 9}-0.022$

37. (DESCRIBE) I am good at verbally conveying my ideas, expectancies and concerns.

Factor 4: Open, non-avoidant orientation

19. (NONAVOID) When I am in pain, I try to avoid the sensation as much as possible.

8. (NONAVOID) I tend to suppress unpleasant feelings and thoughts. Ich neige dazu, unangenehme

Gefühle und Gedanken zu verdrängen.

41. (NONAVOID) I can dwell on unpleasant feelings and sensations. Ich kann bei unangenehmen

Gefühlen und Empfindungen verweilen.

17. (NONIDENTIFY) I observe how my thoughts and feelings come and go.

24. (OBSERVE) I consciously notice everyday sounds, for example, the mowing of the lawn, the ticking of clocks or the sound of a keyboard.

$\begin{array}{rrrr}0.107 & 0.056 & -0.101 & \mathbf{0 . 5 7 6} \\ 0.093 & 0.030 & 0.061 & \mathbf{0 . 5 3 1} \\ -0.016 & -0.004 & 0.141 & \mathbf{0 . 5 2 1} \\ & & & \\ 0.212 & 0.269 & -0.026 & \mathbf{0 . 4 8 6} \\ -0.075 & 0.171 & 0.145 & \mathbf{0 . 4 7 4}\end{array}$

$N=313$; loadings are from pattern matrix; the questionnaire items are in German, which the authors translated into English; these translations are preliminary

and emotion-regulatory skills were controlled by the FFMQ overall score (Table 6, top). Additionally, partial correlations were calculated controlling each CHIME- $\beta$ subscale for its semantically most associated subscale(s) from the FFMQ (Table 6, bottom). Results support the incremental value of the CHIME- $\beta$ over the FFMQ scores. Particularly the CHIME- $\beta$ overall score and the subscale accepting, nonreactive, insightful orientation showed substantial partial correlations with emotion regulation and, to a lesser extent, with symptom load after controlling for the FFMQ scores. 
Table 4 Fit indices of the models in confirmatory factor analysis

\begin{tabular}{|c|c|c|c|c|c|c|}
\hline Model & $d f$ & $X^{2}$ & $X^{2}$ difference & CFI & SRMR & RMSEA $(90 \% \mathrm{CI})$ \\
\hline \multicolumn{7}{|l|}{ Entire sample $(n=313)$} \\
\hline One-factor model & 350 & $1,783.88 * * *$ & & 0.69 & 0.09 & $0.11(0.11-0.12)$ \\
\hline Four-factor model & 344 & $974.30 * * *$ & & 0.87 & 0.06 & $0.08(0.07-0.08)$ \\
\hline Hierarchical four-factor model & 346 & $985.90 * * *$ & $11.60 * *(d f=2)$ & 0.86 & 0.07 & $0.08(0.07-0.08)$ \\
\hline \multicolumn{7}{|c|}{ Meditation-trained individuals $(n=144)$} \\
\hline One-factor model & 350 & $1,075.23 * * *$ & & 0.67 & 0.10 & $0.12(0.11-0.13)$ \\
\hline Four-factor model & 344 & $648.91 * * *$ & & 0.86 & 0.08 & $0.08(0.07-0.09)$ \\
\hline Hierarchical four-factor mode & 346 & $650.50 * * *$ & $1.59(d f=2)$ & 0.86 & 0.08 & $0.08(0.07-0.09)$ \\
\hline
\end{tabular}

${ }^{*} p<0.05 ;{ }^{* *} p<0.01 ; * * * p<0.001$

Group comparisons showed that, as would be expected, in participants who completed the MBSR training the CHIME- $\beta$ overall was significantly higher at the end (mean $=4.41, \mathrm{SD}=0.60$ ) compared to the beginning of the training $($ mean $=3.96, \mathrm{SD}=0.65, t(143)=11.32, p<0.001)$. In the entire sample, male participants reported significantly higher CHIME- $\beta$ overall scores (mean $=4.13, \mathrm{SD}=0.79, n=$ 114) than women $($ mean $=3.94, \mathrm{SD}=0.72, n=199, t(311)=$ $2.11, p=0.04$ ) although the difference was not conspicuous. The CHIME- $\beta$ overall score was not significantly correlated with the age of participants $(p>0.69)$.

Tables 7 and 8 (above) display the intercorrelations between the four latent factors of mindfulness and the loadings of each latent factor to an overreaching mindfulness variable respectively. All correlations and loadings showed the expected directions and were moderate to strong. Moreover, even if the hierarchical model failed to outperform the correlational four-factor model, all standardized regression weights on the higher-order mindfulness factor were above 0.7 , which suggests that the four factors share a relevant proportion of variance.
Factor Analysis in Meditation-Trained Individuals

The one- and four-factor models from the previous analysis were tested with CFA in meditation-trained individuals ( $n=$ 144). As in the entire sample, the one-factor model fitted poorly (Table 4). The four-factor models again showed good fit, except for the CFI. Nevertheless, modification indices gave a pattern different than that in the entire sample, as they did not indicate the presence of residual correlations between items in the accepting, nonreactive, and insightful orientation factor. Rather, they pointed out residual shared variance between a few items from the present awareness, accepting, nonreactive, and insightful orientation, and open, non-avoidant orientation factors. As these residual correlations did not suggest any clear pattern beyond an interconnectedness between items from different factors, no additional PCA was performed.

Other than in the entire sample, the Chi-square difference between the hierarchical and the non-hierarchical four-factor model was nonsignificant. Thus, the more parsimonious hierarchical model was preferred in the case of data from

Table 5 Criterion, discriminant, and convergent intercorrelations

\begin{tabular}{|c|c|c|c|c|c|}
\hline & Overall CHIME- $\beta$ score & $\begin{array}{l}\text { Accepting, nonreactive, } \\
\text { insightful orientation }\end{array}$ & $\begin{array}{l}\text { Present } \\
\text { awareness }\end{array}$ & $\begin{array}{l}\text { Describing of } \\
\text { experiences }\end{array}$ & $\begin{array}{l}\text { Open, non-avoidant } \\
\text { orientation }\end{array}$ \\
\hline GSI & $-0.46 * * *$ & $-0.52 * * *$ & $-0.31 * * *$ & $-0.28 * * *$ & $-0.20 * *$ \\
\hline ER confront & $0.52 * * *$ & $0.52 * * *$ & $0.29 * * *$ & $0.45^{* * *}$ & $0.35^{* * *}$ \\
\hline ER modification & $0.74 * * *$ & $0.76 * * *$ & $0.53 * * *$ & $0.46^{* * *}$ & $0.49 * * *$ \\
\hline FFMQ overall & $0.88 * * *$ & $0.80^{* * *}$ & $0.65 * * *$ & $0.68 * * *$ & $0.60 * * *$ \\
\hline FFMQ observe & $0.67 * * *$ & $0.46^{* * *}$ & $0.73 * * *$ & $0.35^{* *}$ & $0.64 * * *$ \\
\hline FFMQ describe & $0.65^{* * *}$ & $0.43^{* * *}$ & $0.52 * * *$ & $0.85^{* * *}$ & $0.44 * * *$ \\
\hline FFMQ actaware & $0.66^{* * *}$ & $0.57 * * *$ & $0.65 * * *$ & $0.46^{* * *}$ & $0.35^{* *}$ \\
\hline FFMQ nonjudge & $0.46 * * *$ & $0.60 * * *$ & 0.12 & $0.33 * *$ & $0.25^{*}$ \\
\hline FFMQ nonreact & $0.65^{* * *}$ & $0.76^{* * *}$ & $0.26^{*}$ & $0.42 * * *$ & $0.42 * * *$ \\
\hline
\end{tabular}

$N=313$; GSI General Severity Index (BSI), ER confront readiness to confront distressing situations, ER modif modification

${ }^{*} p<0.05 ; * * p<0.01 ; * * * p<0.001 ;$ two-tailed 
Table 6 Incremental validity: partial correlations controlling for FFMQ scores

\begin{tabular}{|c|c|c|c|c|c|}
\hline & $\begin{array}{l}\text { Overall CHIME- } \beta \\
\text { score }\end{array}$ & $\begin{array}{l}\text { Accepting, nonreactive, } \\
\text { insightful orientation }\end{array}$ & $\begin{array}{l}\text { Present } \\
\text { awareness }\end{array}$ & $\begin{array}{l}\text { Describing of } \\
\text { experiences }\end{array}$ & $\begin{array}{l}\text { Open, non-avoidant } \\
\text { orientation }\end{array}$ \\
\hline \multicolumn{6}{|c|}{ Control variable: FFMQ overall } \\
\hline GSI & -0.19 & $-0.27^{*}$ & 0.01 & 0.09 & -0.14 \\
\hline ER confront & $0.43^{* * *}$ & $0.42 * * *$ & 0.12 & $0.26^{*}$ & 0.04 \\
\hline ER modification & $0.39 * *$ & $0.54 * * *$ & 0.07 & -0.15 & 0.16 \\
\hline \multicolumn{6}{|c|}{ Control variable(s): semantically related FFMQ subscales } \\
\hline GSI & & $-0.23^{\dagger}$ & -0.03 & -0.19 & $-0.24^{\dagger}$ \\
\hline ER confront & & $0.38^{* *}$ & $0.25 *$ & $0.37 * *$ & 0.12 \\
\hline ER modification & & $0.60 * * *$ & 0.00 & 0.19 & 0.13 \\
\hline
\end{tabular}

$N=68$; in the bottom part of the table accepting, nonreactive, insightful orientation was controlled for FFMQ nonjudge and FFMQ nonreact, present awareness for FFMQ actaware and FFMQ observe, describing of experiences for FFMQ describe, and open, non-avoidant orientation for FFMQ observe and FFMQ nonreact

${ }^{\dagger} p<0.10 ; * p<0.05 ; * * p<0.01 ; * * * p<0.001$

meditation-trained individuals. Correlations between the latent factors and standardized regression coefficients on the higher-level mindfulness factor are displayed in Tables 5 and 6 (bottom) respectively. The Fisher test for differences between correlations showed that two inter-factor correlations (open, non-avoidant orientation with both accepting, nonreactive, and insightful orientation, and present awareness) were significantly higher $(p<0.05)$ in meditationtrained individuals. The correlation between describing of experiences and accepting, nonreactive, and insightful orientation showed a decreasing tendency in meditationtrained individuals $(p<0.10)$. After Bonferroni correction for six tests, only the correlation between present awareness and open, non-avoidant orientation remained significantly higher $(p<0.008)$. As in the complete sample, but more markedly, describing of experiences showed the lowest regression coefficient to the higher-level mindfulness factor, while open, non-avoidant orientation had the highest regression coefficient (n.b., standardized coefficients above one can occur when factors are not orthogonal; see Jöreskog 1999).

Table 7 Correlations between the four latent mindfulness factors

\section{Discussion}

The aim of this study was to contribute to the development of a self-report scale that is suitable for the assessment of mindfulness as a quasi-trait in a general population, taking into account the scientific literature on mindfulness, current mindfulness scales, and empirical results. For this purpose, a review of the aspects of mindfulness assessed by the available validated mindfulness scales was performed. Nine mindfulness aspects were identified. This was helpful because it provided a common language across the current questionnaires and conceptualizations. A first version of a mindfulness scale that covers the nine identified aspects in a balanced way was constructed.

In a sample largely untrained in meditation, PCA suggested that four mindfulness factors underlie the nine aspects assessed. One factor, present awareness, clearly taps into the first component of mindfulness - self-regulation of attention-of Bishop and colleagues (2004); this factor involves both the awareness of perceptual and bodily experiences and the non-distracted, attentive way of carrying out

\begin{tabular}{|c|c|c|c|}
\hline & Present awareness & Describing of experiences & Open, non-avoidant orientation \\
\hline \multicolumn{4}{|l|}{ Entire sample $(n=313)$} \\
\hline Accepting, nonreactive, insightful orientation & $0.60^{* * *}$ & $0.63^{* * *}$ & $0.72 * * *$ \\
\hline Present awareness & & $0.47 * * *$ & $0.75 * * *$ \\
\hline Describing of experiences & & & $0.60 * * *$ \\
\hline \multicolumn{4}{|l|}{ Mediation-trained individuals $(n=144)$} \\
\hline Accepting, nonreactive, insightful orientation & $0.62 * * *$ & $0.52 * * *$ & $0.81 * * *$ \\
\hline Present awareness & & $0.45^{* * *}$ & $0.85 * * *$ \\
\hline Describing of experiences & & & $0.61 * * *$ \\
\hline
\end{tabular}

$* p<0.05 ; * * p<0.01 ; * * * p<0.001 ;$ two-tailed 
Table 8 Standardized regression weights on the overall mindfulness construct

\begin{tabular}{lllll}
\hline & $\begin{array}{l}\text { Accepting, nonreactive, } \\
\text { insightful orientation }\end{array}$ & $\begin{array}{l}\text { Present } \\
\text { awareness }\end{array}$ & $\begin{array}{l}\text { Describing of } \\
\text { experiences }\end{array}$ & $\begin{array}{l}\text { Open, non-avoidant } \\
\text { orientation }\end{array}$ \\
\hline Entire sample $(n=313)$ & $0.83^{* * *}$ & $0.74 * * *$ & $0.71^{* * *}$ & $0.89^{* * *}$ \\
Meditation-trained individuals $(n=144)$ & $0.79^{* * *}$ & $0.80^{* * *}$ & $0.60^{* * *}$ & $1.04 * * *$ \\
\hline
\end{tabular}

${ }^{*} p<0.05 ; * * p<0.01 ; * * * p<0.001 ;$ two-tailed

everyday activities. Two factors captured a mindful orientation towards experiences: accepting, nonreactive, and insightful orientation and open, non-avoidant orientation. Finally, the fourth factor that emerged from the analysis was describing of experiences. The CHIME- $\beta$ and its factors showed acceptable internal consistencies and generated the expected patterns in criterion, discriminant and convergent validity analyses. Moreover, results suggest that the CHIME- $\beta$ provides incremental value over another mindfulness measure, the FFMQ. In fact, the CHIME- $\beta$ includes aspects of mindfulness such as self-acceptance, an insightful orientation, non-identification with inner experiences and non-avoidance, which are not included in the FFMQ. Prepost comparisons in participants who completed the MBSR intervention showed the expected pattern of heightened reported mindfulness at the end of the intervention. Unexpectedly, male participants showed significantly higher mindfulness scores than women, although the difference was not conspicuous. A similar result was reported in the validation study of the CAM-R (Feldman et al. 2007). In CFA, the four-factor structure yielded comparable results in individuals who were more experienced in meditation, yielding additional supportive evidence for this structure.

Interestingly, a factor addressing a lack of experiential avoidance (vs. a heightened willingness to be exposed to negative situations) and another factor that addresses an accepting stance towards one's own thoughts, feelings, and oneself emerged from the analyses. This points to a meaningful distinction between "acceptance" as conceptualized in the PHLMS (i.e., as lack of experiential avoidance) and acceptance as conceptualized in most other mindfulness questionnaires (i.e., as self-acceptance and nonjudgment). Open, nonavoidant orientation showed the highest correlations with the other factors as well as the highest loading to a putative higher-order mindfulness construct. This supports the particular relevance of this aspect. In fact, a certain degree of openness and willingness to stay in contact with experiences may be viewed as an essential attitude in the development of mindfulness (cf., Hayes and Feldman 2004). The fourth factor of the analysis, describing of experiences, was the only factor exclusively containing items from one of the nine suggested mindfulness aspects. Thus, while eight of the nine suggested mindfulness aspects showed a high degree of interconnectedness, items related to the capacity to put experiences into words appear to denote a capacity that is semantically distinct. From a theoretical point of view, describing own experiences neither belongs to self-regulation of attention nor describes an orientation towards experiences. Most definitions of mindfulness do not include this aspect (Bishop et al. 2004; Kabat-Zinn 1994). In the mindfulness tradition, labeling of experiences is often considered a component of mindfulness meditation. However, traditionally, such labeling is typically not an accurate description of emotions or of the contents of thought; more commonly, it is a general recognition that thoughts are thoughts, feelings are feelings, etc. This does not involve categorization, reflection, or introspection (Brown et al. 2007; Gunaratana 2002). In accordance with this, among the four factors, describing of experiences showed the weakest regression coefficient to a putative higher-order mindfulness construct and the weakest correlations to the other three factors. Moreover, describing of experiences showed a tendency for decreasing associations with other mindfulness factors in meditation-trained individuals. It is thus unclear to what extent the ability to verbally describe experiences as measured by the KIMS or FFMQ constitutes a component of mindfulness and should thus be included in a parsimonious assessment of mindfulness.

In sum, with reference to the first open question described above concerning an appropriate conceptual coverage of mindfulness scales, the present results suggest a conceptualization of mindfulness as comprising one factor covering the self-regulation of attention component as described by Bishop and colleagues (2004) and at least two factors covering a mindful orientation: one describing a selfaccepting, nonjudgmental, insightful, and non-reactive stance towards experiences and the other describing an open, non-avoidant presence. Results suggest that an open, non-avoidant presence may be viewed as a fundamental attitude in mindfulness, while describing of experiences may be a capacity that is related to mindfulness rather than constituting an aspect of mindfulness.

In the CFA, the four-factor model showed a slight lack of fit in both the entire sample and in meditation-trained individuals. Nevertheless, the reason for this slight lack of fit was different in the two analyses: in the entire sample, the model failed to account for a tendency of the items capturing a nonjudgmental stance to form a factor of their own within the accepting, nonreactive, and insightful orientation factor. 
In fact, the accepting, nonreactive, and insightful orientation factor tended to split into two distinct aspects, one uniformly addressing nonjudgment towards experiences and the other comprising self-acceptance, an open and insightful orientation, and non-reactivity. This tendency may be due to the different meaning of the items. Alternatively, it may be a consequence of the negative formulation of the nonjudgment items, which contrasts with the positive formulation of all other items within the accepting, nonreactive, and insightful orientation factor. On the other hand, in meditation-trained individuals, the slight lack of fit of the model was due to associations between factors (vs. withinfactor) that were not accounted for in the model. In other words, in this sample the model failed to account for associations between items from different factors. This result suggests that with increasing experience in mindfulness meditation, the different aspects of the construct become more interconnected. This is also supported by the fact that only in meditation-trained individuals, the model assuming a higher-level mindfulness factor was clearly superior to a model in which the four factors were merely correlated. Moreover, inter-factor correlations, excluding those involving describing of experiences, tended to be higher in meditation-trained individuals than in the entire sample. This is a relevant finding with reference to the second issue concerning the kind of relationship among mindfulness aspects and, in particular, the issue about the possibility to discern distinct aspects of mindfulness. An important research issue is the need to measure complex constructs at the component level, which allows testing hypotheses about their relationships with other variables (Hough and Schneider 1995; Smith et al. 2003; Smith and McCarthy 1995). The current results suggest the possibility of a stable solution over different populations. Nevertheless, the results also suggest that the relationship between the aspects of mindfulness may tend towards one involving heightened interconnectedness through experience with mindfulness meditation and a growing understanding of the construct. This may help explain the current inconsistency in findings in the scientific literature, i.e., the fact that some findings support a multifaceted conception of mindfulness (Baer et al. 2006; Cardaciotto et al. 2008; Lau et al. 2006, Leary and Tate 2007) while others indicate a more holistic conceptualization of mindfulness (Buchheld et al. 2001; Chadwick et al. 2008; Feldman et al. 2007). The current results differ in several ways from the findings of a similar study that led to the construction of the FFMQ (Baer et al. 2006). In the study by Baer et al., all items of five mindfulness questionnaires were subjected to EFA, yielding five interpretable factors: nonreact, observe, actaware, describe, and nonjudge. The main reason for the different factorizations may be that some aspects of mindfulness were represented to a greater extent than others in terms of quantity of items and that the structure of the FFMQ was strongly influenced by the structure of the KIMS, which was the longest scale included in the EFA. This was not the case with the present study, as particular attention was paid to address every aspect of mindfulness with an equal number of items.

It should be noted that the current study has a number of limitations. About a quarter of the participants reported practicing meditation regularly. Meditation practice, however, was defined in a broad sense, and included Tai Chi, Yoga, Qui Gong, and relaxation techniques. Moreover, the difference in meditation experience between the two groups consisted of an 8-week mindfulness-based intervention and was thus limited. Nevertheless, as MBSR constituted a rather intensive intervention, involving daily formal and informal mindfulness practice, as well as weekly meetings and theoretical information, important changes related to mindfulness practice may well be expected. Third, the present handling of missing data may be suboptimal as it was influenced by the capabilities of the software used. Nevertheless, given the small percentage of missing values in the current data the chosen procedure should not have sensibly influenced the results. Fourth, the arrangement of aspects of mindfulness proposed in the present study is possibly only one of several different meaningful classifications and it may be expected that results from a questionnaire based on another arrangement may have yielded different results. Finally, the quality of the questionnaire may have been improved by experts' ratings of the items as well as by the inclusion of more items per mindfulness aspect. Nevertheless, according to Fabrigar et al. (1999), three to five items per expected factor should provide an adequate item pool.

The current study also has substantial merits. The item pool included all aspects of mindfulness that have been proposed in previous operationalizations. Moreover, biases that may have arisen from an unequal inclusion of these aspects were avoided. The study involved a comparatively large sample and implemented sophisticated statistical methods. Finally, the results help to explain some of the inconsistencies of previous findings and provide clear developments for the conceptualization and operationalization of mindfulness.

Future research should further analyze the influence of meditation practice on the structure of mindfulness in order to better characterize this construct and provide measures of mindfulness that are adequate for different populations. Particular attention should be paid to finding a middle ground between measuring mindfulness at the aspect level and avoiding giving the construct a factitious structure. Furthermore, the measurement of mindfulness using methods other than self-report deserves greater attention. In summary, the findings reported here have helped to shed light on the mindfulness construct. The results offer important directions for the construction of the final version of the CHIME. 


\section{References}

Acock, A. C. (2005). Working with missing values. Journal of Marriage and Family, 67, 1012-1028.

Baer, R. (Ed.). (2010). Assessing mindfulness and acceptance processes in clients: Illuminating the theory and practice of change. Oakland: Context Press/New Harbinger.

Baer, R. A., Smith, G. T., \& Allen, K. B. (2004). Assessment of mindfulness by self-report: the Kentucky inventory of mindfulness skills. Assessment, 11, 191-206.

Baer, R. A., Smith, G. T., Hopkins, J., Krietemeyer, J., \& Toney, L. (2006). Using self-report assessment methods to explore facets of mindfulness. Assessment, 13, 27-45.

Bandalos, D. L., \& Finney, S. J. (2001). Item parceling issues in structural equation modeling (pp. 269-296). In G. A. Marcoulides, \& R. E. Schumacker (Eds.), New developments and techniques in structural equation modeling (pp. 269-296). Hillsdale, NJ: Erlbaum.

Bergomi, C. (2007). Achtsamkeit, buddhistische Meditation und psychische Gesundheit (Unpublished master's thesis). Psychology Department, University of Bern, Switzerland. Retrieved from http://www.upd.unibe.ch/research/papers/Liz_Bergomi.pdf.

Berking, M., \& Znoj, H. (2008). Entwicklung und Validierung eines Fragebogens zur standardisierten Selbsteinschätzung emotionaler Kompetenzen (SEK-27). Zeitschrift für Psychiatrie, Psychologie und Psychotherapie, 56, 141-152.

Berking, M., Wupperman, P., Reichardt, A., Pejic, T., Dippel, A., \& Znoj, H. (2008). General emotion-regulation skills as a treatment target in psychotherapy. Behavior Research and Therapy, 46, $1230-1237$.

Bishop, S. R., Lau, M., Shapiro, S., Carlson, L., Anderson, N. D., Carmody, J., et al. (2004). Mindfulness: a proposed operational definition. Clinical Psychology: Science and Practice, 11, 230 241.

Brown, T. A. (2006). Confirmatory factor analysis for applied research. New York: Guilford.

Brown, K. W., \& Ryan, R. M. (2003). The benefits of being present: mindfulness and its role in psychological well-being. Journal of Personality and Social Psychology, 84, 822-848.

Brown, K. W., \& Ryan, R. M. (2004). Perils and promises in defining and measuring mindfulness: observations from experience. Journal of Clinical Psychology, Science \& Practice, 11, 242-248.

Brown, K. W., Ryan, R. M., \& Creswell, J. D. (2007). Mindfulness: theoretical foundations and evidence for its salutary effects. Psychological Inquiry, 18, 211-237.

Brown, K. W., West, A. M., Loverich, T. M., \& Biegel, G. M. (2011). Assessing adolescent mindfulness: Validation of an adapted mindful attention awareness scale in adolescent normative and psychiatric populations. Psychological Assessment, in press: Epub ahead of print retrieved May 10, 2011, doi: 10.1037/a0021338.

Browne, M. W., \& Cudeck, R. (1993). Alternative ways of assessing model fit. In K. A. Bollen \& J. S. Long (Eds.), Testing structural equation models (pp. 136-162). Newbury Park: Sage.

Buchheld, N., Grossman, P., \& Walach, H. (2001). Measuring mindfulness in insight meditation (Vipassana) and meditation-based psychotherapy: the development of the Freiburg Mindfulness Inventory (FMI). Journal for Meditation and Meditation Research, 1, 11-34.

Burg, J., \& Michalak, J. (2010). Assessing mindfulness: Is there an alternative to questionnaires? Talk given at the XL European Association for Behavioural and Cognitive Therapies Congress, Milan, Italy.

Cardaciotto, L. (2005). Assessing mindfulness: The development of a bi-dimensional measure of awareness and acceptance. Unpublished doctoral dissertation, Drexel University, Philadelphia.
Cardaciotto, L., Herbert, J. D., Forman, E. M., Moitra, E., \& Farrow, V. (2008). The assessment of present-moment awareness and acceptance: the Philadelphia mindfulness scale. Assessment, 15, 204-223.

Cattell, R. B. (1974). Radial item parcel factoring vs. item factoring in defining personality structure in questionnaires: theory and experimental checks. Australian Journal of Psychology, 26, 103-119.

Chadwick, P., Hember, M., Symes, J., Peters, E., Kuipers, E., \& Dagnan, D. (2008). Responding mindfully to unpleasant thoughts and images: reliability and validity of the Southampton Mindfulness Questionnaire (SMQ). British Journal of Clinical Psychology, 47, 451-455.

Christopher, M. S., Charoensuk, S., Gilbert, B. D., Neary, T. J., \& Pearce, K. L. (2009). Mindfulness in Thailand and the United States: a case of apples versus oranges? Journal of Clinical Psychology, 65, 590-612.

Coffey, K. A., \& Hartman, M. (2008). Mechanisms of action in the inverse relationship between mindfulness and psychological distress. Complementary Health Practice Review, 13, 79-91.

Collins, S. E., Chwala, N., Hsu, S. H., Grow, J., Otto, J. M., \& Marlatt, G. A. (2009). Language-based measures of mindfulness: initial validity and clinical utility. Psychology of Addictive Behaviors, $23,743-749$.

Costello, A. B., \& Osborne, J. W. (2005). Best practices in exploratory factor analysis: four recommendations for getting the most from your analysis, Practical Assessment, Research \& Evaluation: A Pear-reviewed Electronic Journal, 10, 1-9. Retrieved from http:// pareonline.net/pdf/v10n7.pdf.

Cudeck, R., \& Browne, M. W. (1983). Cross-validation of covariance structures. Multivariate Behavioral Research, 18, 147-167.

Dimidjian, S., \& Linehan, M. M. (2003). Defining an agenda for future research on the clinical application of mindfulness practice. Clinical Psychology: Science and Practice, 10, 166-171.

Dobkin, P. L. (2008). Mindfulness-based stress reduction: what processes are at work? Complementary Therapies in Clinical Practice, $14,8-16$.

Everitt, B. S. (2002). The Cambridge Dictionary of Statistics, 2nd Edition, CUP.

Fabrigar, L. R., Wegener, D. T., MacCallum, R. C., \& Strahan, E. J. (1999). Evaluating the use of exploratory factor analysis in psychological research. Psychological Methods, 4, 272-299.

Feldman, G. C., Hayes, A. M., Kumar, S. M., Greeson, J. G., \& Laurenceau, J. P. (2007). Mindfulness and emotion regulation: the development and initial validation of the cognitive and affective mindfulness scale-revised (CAMS-R). Journal of Psychopathology and Behavioral Assessment, 29, 177-190.

Franke, G. H. (2000). Brief symptom inventory (BSI). Göttingen: Beltz.

Frewen, P. A., Evans, El M., Maraj, N., Dozois, D. J. A., \& Partridge, K. (2008). Letting go: mindfulness and negative automatic thinking. Cognitive Therapy and Research, 32, 758-774.

Gosling, S. D., Vazire, S., Srivastava, S., \& John, O. P. (2004). Should we trust web-based studies? A comparative analysis of six preconceptions about Internet questionnaires. American Psychologist, 59, 93-104.

Graham, J. W. (2009). Missing data analysis: making it work in the real world. Annual Review of Psychology, 60, 549-576.

Greco, L. A., Baer, R. A., \& Smith, G. T. (2011). Assessing mindfulness in children and adolescents: Development and validation of the child and adolescent mindfulness measure (CAMM). Psychological Assessment, in press: Epub ahead of print retrieved May 10, 2011, doi: 10.1037/a0022819.

Grossman, P., Niemann, L., Schmidt, S., \& Walach, H. (2004). Mindfulness-based stress reduction and health benefits: a metaanalysis. Journal of Psychosomatic Research, 57, 35-43.

Gunaratana, B. H. (2002). Mindfulness in plain English. Somerville: Wisdom. 
Hayes, A. M., \& Feldman, G. (2004). Clarifying the construct of mindfulness in the context of emotion regulation and the process of change in therapy. Clinical Psychology: Science and Practice, $11,255-262$.

Hayes, S. C., \& Wilson, K. G. (2003). Mindfulness: method and process. Clinical Psychology: Science and Practice, 10, 161-165.

Hayes, S. C., Wilson, K. G., Gifford, E. V., Follette, V. M., \& Strosahl, K. D. (1996). Experiential avoidance and behavioral disorders: a functional dimensional approach to diagnosis and treatment. Journal of Consulting and Clinical Psychology, 64, 1152-1168.

Heidenreich, T., Zarbock, G., Ströhle, G., \& Michalak, J. (September, 2011). The German version of the five-facet mindfulness questionnnaire (FFMQ): Psychometric properties and relationship with the Schema Mode Inventory. Talk given at the 41st European Association for Behavioural and Cognitive Therapies Congress, Reykjavík, Iceland.

Hofmann, S. G., Sawyer, A. T., Witt, A. A., \& Oh, D. (2010). The effect of mindfulness-based therapy on anxiety and depression: a meta-analytic review. Journal of Consulting and Clinical Psychology, 78, 169-183.

Hough, L. M., \& Schneider, R. J. (1995). Personality traits, taxonomies, and applications in organizations. In K. R. Murphy (Ed.), Individuals and behavior in organizations (pp. 31-88). San Francisco: Jossey-Bass.

John, O. P., \& Benet-Martinez, V. (2000). Measurement: reliability, construct validation, and scale construction. In H. T. Reis \&C. M. Judd (Eds.), Handbook of research methods in social and personality psychology (pp. 339-369). New York: Cambridge.

Jolliffe, I. T. (2002). Principal component analysis, Springer series in statistics (2nd ed.). New York: Springer.

Jöreskog, K. G. (1999). How large can a standardized coefficient be? Lincolnwood, IL: Scientific Software International. Available at www.ssicentral.com.

Kabat-Zinn, J. (1990). Full catastrophe living: using the wisdom of your body and mind to face stress, pain, and illness. New York: Dell.

Kabat-Zinn, J. (1994). Wherever you go, there you are: mindfulness meditation in everyday life. New York: Hyperion.

Kline, R. B. (2005). Principles and practice of structural equation modeling. New York: Guilford.

Lau, M., Bishop, S. R., Segal, Z. V., Buis, T., Anderson, N. D., Carlson, L., et al. (2006). The Toronto mindfulness scale: development and validation. Journal of Clinical Psychology, 62, 1445-1467.

Leary, M. R., \& Tate, E. B. (2007). The multi-faceted nature of mindfulness. Psychological Inquiry, 18(4), 251-255. doi:10.1080/10478400701598355.

Leigh, J., Bowen, S., \& Marlatt, G. A. (2005). Spirituality, mindfulness and substance abuse. Addict Behavior, 30, 1335-1341.

Linehan, M. M. (1993). Cognitive behavioral therapy of borderline personality disorder. New York: Guilford.

MacCallum, R. C., Widaman, K. F., Zhang, S., \& Hong, S. (1999). Sample size in factor analysis. Psychological Methods, 4, 84-99.

Malinowski, P. (2008). Mindfulness as psychological dimension: concepts and applications. Irish Journal of Psychology, 29, 153-164.

Marsh, H. W., Hau, K. T., \& Wen, Z. (2004). In search of golden rules: comment on hypothesis-testing approaches to setting cutoff values for fit indexes and danger in overgeneralizing $\mathrm{Hu}$ and Bentler's (1999) findings. Structural Equation Modeling, 11, 320-341.
Marsh, H. W., Hau, K. T., \& Grayson, D. (2005). Goodness of fit evaluation in structural equation modeling. In Maydeu-Olivares, A. \& McArdle, J. (Eds.), Contemporary Psychometrics: A festschrift to Roderick P. McDonald (pp. 275-340). Mahwah, NJ: Lawrence Erlbaum Associates, Inc.

O'Connor, B. P. (2000). SPSS and SAS programs for determining the number of components using parallel analysis and Velicer's MAP test. Behavior Research Methods, Instruments, \& Computers, 32, 396-402.

Park, H. S., Dailey, R., \& Lemus, D. (2002). The use of exploratory factor analysis and principal components analysis in communication research. Human Communication Research, 28, 562-577.

Ryff, C. D., \& Keyes, C. L. M. (1995). The structure of psychological well-being revisited. Journal of Personality and Social Psychology, 69, 719-727.

Schermelleh-Engel, K., Moosbrugger, H., \& Müller, H. (2003). Evaluating the fit of structural equation models: tests of significance and descriptive goodness-of-fit measures. Methods of Psychological Research Online, 8, 23-76.

Segal, Z. V., Williams, J. M. G., \& Teasdale, J. D. (2002). Mindfulnessbased cognitive therapy for depression: a new approach to preventing relapse. New York: Guilford.

Shapiro, S. L., Carlson, L., Astin, J., \& Freedman, B. (2006). Mechanisms of mindfulness. Journal of Clinical Psychology, 72, 373-36.

Shigaki, C. L., Glass, B., \& Schopp, L. H. (2006). Mindfulness-based stress reduction in medical settings. Journal of Clinical Psychology in Medical Settings, 13, 209-216.

Smith, G. T., \& McCarthy, D. M. (1995). Methodological considerations in the refinement of clinical assessment instruments. Psychological Assessment, 7, 300-308.

Smith, G. T., Fischer, S., \& Fister, S. M. (2003). Incremental validity principles in test construction. Psychological Assessment, 15, 467-477.

Teasdale, J. D. (1999). Metacognition, mindfulness and modification of mood disorders. Clinical Psychology \& Psychotherapy, 5, 146-155.

Teasdale, J. D., Segal, Z. V., \& Williams, J. M. G. (1995). How does cognitive therapy prevent relapse and why should attentional control (mindfulness) training help? Behaviour Research and Therapy, 33, 25-39.

Thompson, B. L., \& Waltz, J. (2007). Everyday mindfulness and mindfulness meditation: overlapping constructs or not? Personality and Individual Differences, 43, 1875-1885.

Tomarken, A. J., \& Waller, N. G. (2005). Structural equation modeling: strengths, limitations, and misconceptions. Annual Review of Clinical Psychology, 1, 31-65.

Walach, H., Buchheld, N., Buttenmüller, V., Kleinknecht, N., \& Schmidt, S. (2006). Measuring mindfulness - the Freiburg Mindfulness Inventory (FMI). Personality and Individual Differences, 40, 1543-1555.

West, S. G., Finch, J. F., \& Curran, P. J. (1995). Structural equation models with nonnormal variables. In R. H. Hoyle (Ed.), Structural equation modeling: concepts, issues, and applications (pp. 5775). Thousand Oaks: Sage.

Zwick, W. R., \& Velicer, W. F. (1986). Factors influencing five rules for determining the number of components to retain. Psychological Bulletin, 99, 432-442. 